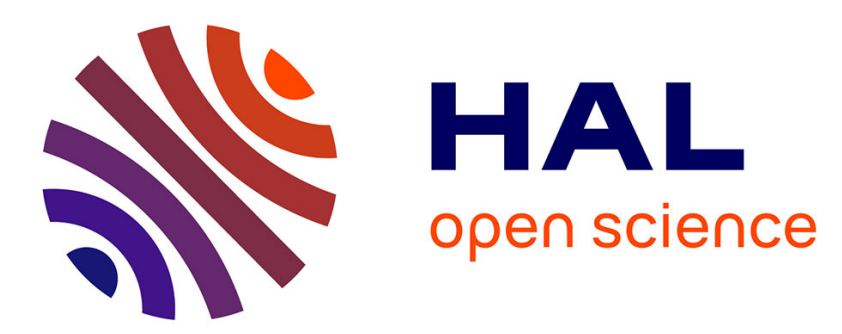

\title{
Différenciation des cellules souches embryonnaires humaines en cellules épithéliales respiratoires
}

\author{
A. Navarre, D. Adam, E. Luczka, P. Birembaut, C. Coraux
}

\section{To cite this version:}

A. Navarre, D. Adam, E. Luczka, P. Birembaut, C. Coraux. Différenciation des cellules souches embryonnaires humaines en cellules épithéliales respiratoires. Journées de Recherche Respiratoire (J2R), Oct 2014, Bordeaux, France. pp.335, 10.1016/j.rmr.2015.02.076 . hal-03011863

\section{HAL Id: hal-03011863 \\ https://hal.univ-reims.fr/hal-03011863}

Submitted on 23 Nov 2020

HAL is a multi-disciplinary open access archive for the deposit and dissemination of scientific research documents, whether they are published or not. The documents may come from teaching and research institutions in France or abroad, or from public or private research centers.
L'archive ouverte pluridisciplinaire HAL, est destinée au dépôt et à la diffusion de documents scientifiques de niveau recherche, publiés ou non, émanant des établissements d'enseignement et de recherche français ou étrangers, des laboratoires publics ou privés. 


\title{
Différenciation des cellules souches embryonnaires humaines en cellules épithéliales respiratoires
}

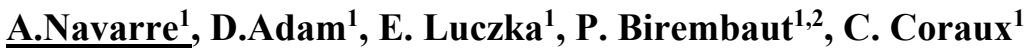 \\ ${ }^{1}$ Inserm UMR-S 903, CHU Maison Blanche, SFR CAP-SANTE, URCA, Reims, France \\ ${ }^{2}$ CHU Maison Blanche, Laboratoire Pol Bouin, Reims
}

Introduction : La mucoviscidose $(\mathrm{CF})$ est la maladie génétique la plus fréquente. Les perspectives de thérapie prorégénératrice, cellulaire et/ou génique sont nombreuses dans la $\mathrm{CF}$. La production in vitro d'épithélium respiratoire non-CF mais surtout $\mathrm{CF}$, en grande quantité pourrait fournir un modèle pré-clinique humain pour le criblage de molécules thérapeutiques. Le but de la présente étude est d'obtenir, à partir de cellules souches embryonnaires humaines (hES), des progéniteurs épithéliaux en préambule à leur différenciation en cellules épithéliales respiratoires.

Méthodes : Les cellules hES (lignée Sahlgrenska 1 (SA-O1)), sont des cellules provenant d'un embryon surnuméraire issu de fécondation in vitro et ne faisant plus l'objet d'un projet parental. Ces cellules sont cultivées sur des cellules nourricières dont la prolifération est stoppée par un traitement à la mitomycine $\mathrm{C}(\mathrm{STO})$ ou sur des substrats tels que le matrigel et la fibronectine plasmatique, dans un milieu commercial optimisé pour les cellules épithéliales respiratoires (BEGM) en présence de $0,5 \mathrm{nM}$ de BMP4 et de $0,3 \mathrm{mM}$ d'acide ascorbique (AA) ou rétinoïque (AR) en continu, ou pendant 3 jours ou 6 jours. La présence de cellules basales progénitrices épithéliales (CK13 positives) a été examinée par détection immunocytochimique.

Résultats : Nos résultats montrent par immunocytochimie la présence de cellules CK13 positives dès 20 jours de traitement continu AA/BMP4 sur cellules STO. Sur matrigel, nous observons une forte présence de cellules exprimant la CK13 dès 30 jours de culture avec 6 jours de traitement AA/BMP4. Ce pourcentage diminue avec 3 jours de traitement AA/BMP4 et s'approche de zéro en condition de traitement AR/BMP4. Après 6 jours de traitement AA/BMP4, la fibronectine plasmatique semble être un substrat moins efficace que les cellules STO ou le matrigel pour l'induction de la différenciation des cellules CK13 positives.

Conclusion : Il apparait qu'une stimulation de 6 jours avec la combinaison AA/BMP4 sur matrigel soit suffisante pour induire la différenciation efficace des cellules hES en cellules basales épithéliales. Il apparait également que l'AR soit un inducteur moins puissant que l'AA, et que les cellules hES se développent mieux sur le matrigel que sur la fibronectine plasmatique. Il sera intéressant par la suite de discriminer un ou plusieurs marqueurs membranaires permettant d'isoler les cellules d'intérêt CK13 positives par cytométrie en flux afin de les amplifier spécifiquement. Le devenir de ces cellules pourra alors être évalué in vitro, in vivo ou ex vivo afin de déterminer leur capacité à reconstituer un épithélium complet, mature et fonctionnel.

Mot-clé : Mucoviscidose, cellules souches embryonnaires humaines, différenciation, progéniteurs épithéliaux. 